\title{
Template-free nanoporous cellulose films on Silicon substrates
}

\author{
Carapeto, A.P., Ferraria, A.M. , Brogueira, P.* , Boufi, S. .** and Botelho do Rego, A.M.
}

* Centro de Química-Física Molecular and IN, Instituto Superior Técnico, University of Lisbon, Av. Rovisco Pais, 1049-001 Lisboa, PORTUGAL

*** ICEMS, Department of Physics, Instituto Superior Técnico, University of Lisbon, Av. Rovisco Pais, 1049001 Lisboa, PORTUGAL.

${ }^{* * *}$ Laboratoire des Sciences des Matériaux et Environnement, University of Sfax, Route Soukra km 3.5 - BP $\mathrm{n}^{\circ} 1171$ - 3000 Sfax, TUNISIA.

Email: ana.ferraria@tecnico.ulisboa.pt

Nanoporous materials have applications in multidisciplinary fields. Ideal nanoporous materials have a high surface to volume ratio due to a large porosity, with very ordered and uniform pore structure, allied to high stability and durability [1]. The large internal surface area of nanoporous materials favours their use as functional structures that can interact with different atoms, ions or molecules. In biosensing applications [2], a high porosity degree enables the immobilization of a higher percentage of molecules or particles. Nanoporous materials can be synthesized from polymeric materials which are widely used in biological applications and nanotechnology. Among biopolymer structures, cellulose films are very interesting since cellulose is the most abundant polymer in biosphere. It has intrinsic physico-chemical properties, such as biodegradability and biocompatibility, which are key characteristics for cell and tissue engineering [3] and can be easily modified with different functional groups suitable to biosensing applications.

However, it is difficult to cast cellulose thin films because of cellulose insolubility in conventional solvents. The strategy used until now is to partially silylate cellulose to transform it into trimethylsilyl cellulose (TMSC), a derivative soluble in volatile solvents, ( and after spreading into a film, using the conventional methods, cellulose is regenerated (CellR) by hydrolyzing the TMSC under $\mathrm{HCl}$ vapours.

In this work, we report the preparation of ultrathin submicro- and nanoporous cellulose films onto Si (100). The effect of different experimental conditions of preparation on the film surface morphology was studied, namely the role of the film casting method (spin- vs. dip-coating), solvent (toluene or THF) and substrate pre-treatment (hydrophilicity degree).

The surface morphological structures presented in this work were never obtained before for cellulose films without the use of templates. A rather regular 2D pore network was obtained onto the less hydrophilic Si substrate (contact angle $\cong 68^{\circ}$ ), after two consecutive dips (with an intercalary rotation of $180^{\circ}$ ) in TMSC diluted in toluene and regeneration at the end. With this procedure, pores are grown directly and spontaneously on cellulose films during the regeneration process, without using templates. All the surfaces were characterized by atomic force microscopy (AFM).The application of these innovative structures seems to be very promising in different areas of biomedicine (biosensors) or electronics.

The authors acknowledge FCT for A. P. Carapeto Grant SFRH/BD/75734/2011 and strategic project PEstOE/CTM/LA0024/2011.

[1] Lu, G. Q. and Zhao, X. S., Nanoporous Materials: Science and Engineering, ICP, 2004.

[2] Martin, C.R. et al., Science 317, 331-332, 2007.

[3] Alila, S. et al., Carbohydrate Polymers 77, 553-562, 2009. 


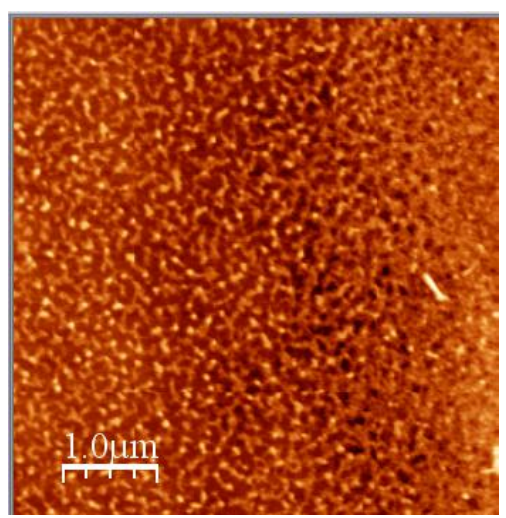

a)
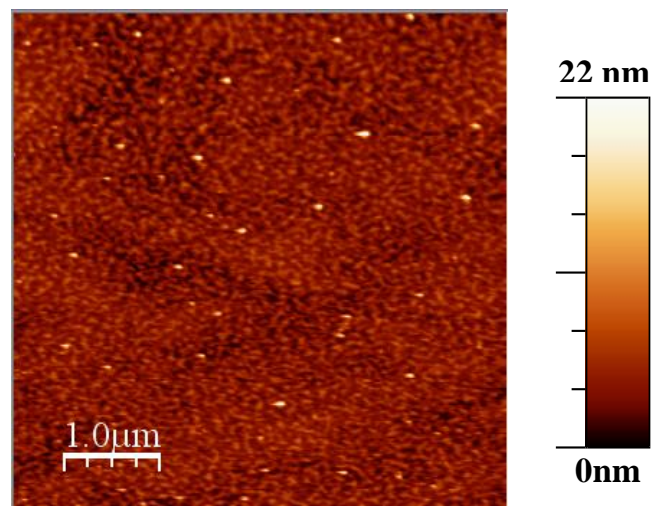

b)

Figure 1. AFM results for the less hydrophilic silicon substrates, dip-coated with TMSC films issued from TMSC solutions in toluene: a) one dip and b) two dips.

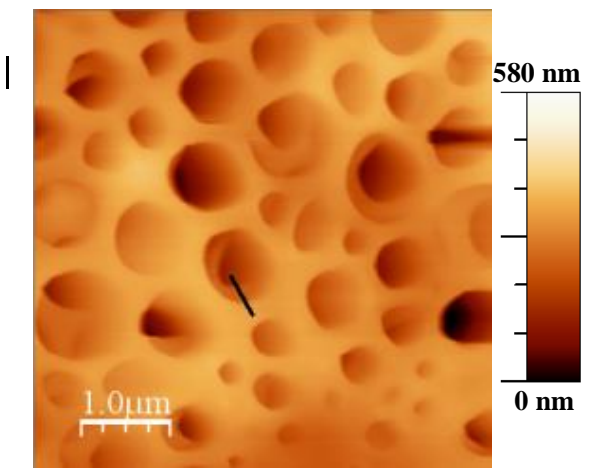

a)

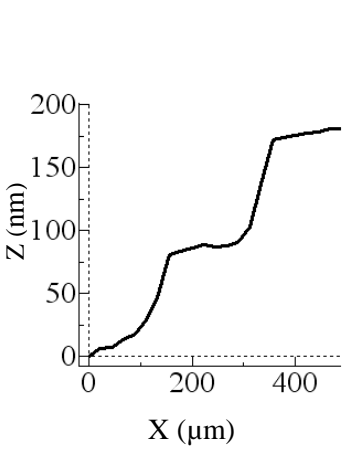

b)

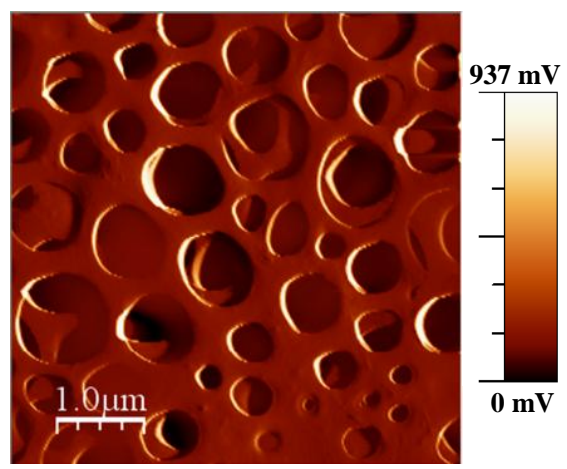

c)

Figure 2. AFM results for the less hydrophilic silicon substrate after two consecutive dips in TMSC diluted in toluene and regeneration at the end. a) topographic image, b) profile, c) phase image.

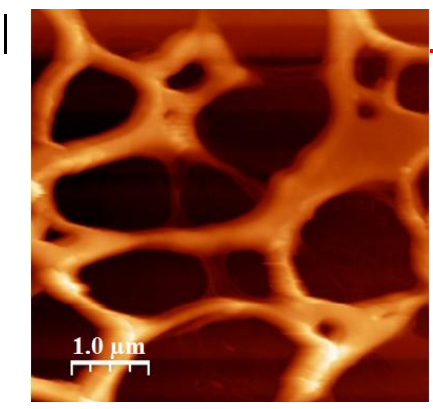

a)

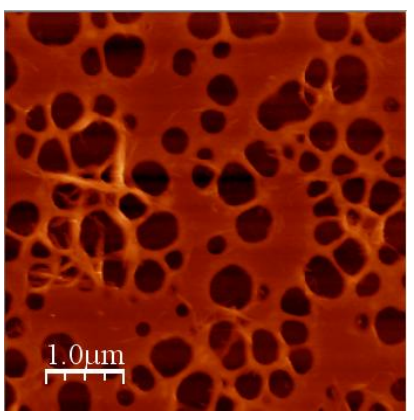

b)
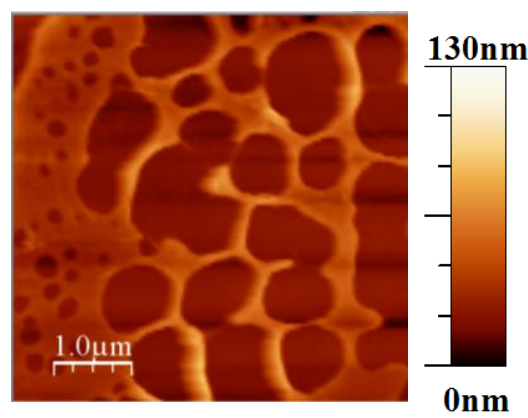

c)

Figure 3. AFM results for a) the more hydrophilic silicon substrate after two consecutive dips in TMSC diluted in toluene and regeneration at the end, b) the less hydrophilic silicon substrate after two consecutive dips in TMSC diluted in THF and regeneration at the end, c) the less hydrophilic silicon substrate after spin-coating twice in TMSC diluted in toluene and regeneration at the end. 